Network Working Group

Request for Comments: 2143

Category: Experimental

B. Elliston

Compucat Research

May 1997

Encapsulating IP with the Small Computer System Interface

Status of this Memo

This memo defines an Experimental Protocol for the Internet community. This memo does not specify an Internet standard of any kind. Discussion and suggestions for improvement are requested. Distribution of this memo is unlimited.

Table of Contents

1. Introduction . . . . . . . . . . . . . . . . . . . . 1

2. Brief background to the Small Computer System Interface . 2

3. Link Encapsulation . . . . . . . . . . . . . . . . . . 3

4. An Address Resolution Protocol. . . . . . . . . . . . . . 4

5. Scalability .. . . . . . . . . . . . . . . . 4

6. Possible applications . . . . . . . . . . . . . . . 5

7. Security considerations . . . . . . . . . . . . . . 5

8. References . . . . . . . . . . . . . . . . . 5

9. Author's Address . . . . . . . . . . . . . . . 5

1. Introduction

As the capacity of local area networks increases to meet the demands of high volume application data, there is a class of network intensive problems which may be applied to small clusters of workstations with high bandwidth interconnection.

A general observation of networks is that the bit rate of the data path typically decreases as the distance between hosts increases. It is common to see regional networks connected at a rate of $64 \mathrm{Kbps}$ and office networks connected at lo0Mbps, but the inverse is far less common .

The same is true of peripheral and memory interconnection. Memory close to a CPU's core may run at speeds equivalent to a gigabit network. More importantly, devices such as disks may connect a number of metres away from its host at speeds well in excess of current local area network capacity. 
This document outlines a protocol for connecting hosts running the TCP/IP protocol suite over a Small Computer system Interface (SCSI) bus. Despite the limitation in the furthest distance between hosts, SCSI permits close clusters of workstations to communicate between each other at speeds approaching 360 megabits per second.

The proposed introduction of newer SCSI implementations such as serial SCSI will bring much faster communication at greater distances.

2. Background to the Small Computer system Interface (SCSI)

SCSI defines a physical and data link protocol for connecting peripherals to hosts. Devices connect autonomously to a bus and send synchronous or asynchronous messages to other devices.

Devices are identified by a numeric identifier (ID). For the original SCSI protocol, devices are given a user-selectable SCSI ID between 0 and 7 . Wide SCSI specifies a range of SCSI IDs between 0 and 15. The most typical SCSI configuration comprises of a host adapter and one or more SCSI- capable peripherals responding to asynchronous messages from the host adapter.

The most critical aspect of the protocol with respect to its use as a data link for the Internet protocols is that a SCSI device must act as an "initiator" (generator of SCSI commands/requests) or a "target" (a device which responds to SCSI commands from the initiator). This model is correct for a master/slave relationship between host adapter and devices. The only time an initiator receives a message addressed to it is in response to a command issued by it in the past and a target device always generates a response to every command it receives.

Clearly this is unsuitable for the peer-to-peer model required for multiple host adapters to asynchronously send SCSI commands to one another without surplus bus traffic. Furthermore, some host adapters may refuse to accept a message from another adapter as it expects to only initiate SCSI commands. This restriction to the protocol requires that SCSI adapters used for IP encapsulation support what is known as "target mode", with software device driver support to pass these messages up to higher layer modules for processing. 


\section{Link Encapsulation}

The ANSI SCSI standard defines classes of peripherals which may be interconnected with the SCSI protocol. One of these is the class of "communication devices" [1].

The standard defines three message types capable of carrying a general-purpose payload across communication devices. Each of these are known as the "SEND MESSAGE" message type, but the size and and structure of the message header differs amongst them. The three forms of message header are six (6), ten (10) and twelve (12) bytes long.

It was decided that the ten byte header offers the greatest flexibility for encapsulating version 4 IP datagrams for the following reasons:

a. The transfer length field is 16 bits in size which is perfectly matched to the datagram length field in IP version 4. Implementations of IP will run efficiently as datagrams will never be fragmented over SCSI networks.

b. The SCSI "stream select" field, which was designed to permit a device to specify the stream of data to which a block belongs, may be used to encode the payload type (in a similar manner to the Ethernet frame type field). For consistency, the lowest four bits of the "stream select" field should match the set of values assigned by the IEEE for Ethernet protocol types.

Encapsulating an IP datagram within a SCSI message is straightforward:

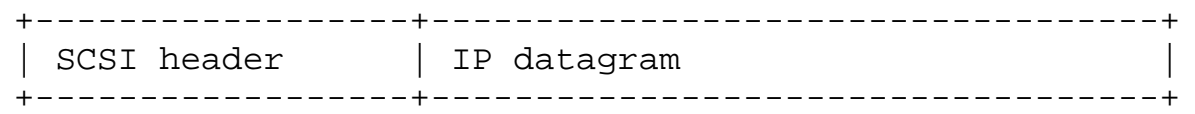

The fields of the SCSI header should be completed as follows:

$\begin{array}{lll}\text { Byte } 0: & \text { 0x2A (SEND_MESSAGE (10) opcode) } \\ \text { Byte 1: } & \text { Logical unit number encoded into top } 3 \text { bits } \mid \text { 0x00 } \\ \text { Byte 2: } & \text { 0x00 } \\ \text { Byte 3: } & \text { 0x00 } \\ \text { Byte 4: } & 0 \times 00 \\ \text { Byte 5: } & \text { Protocol type encoded into lowest } 4 \text { bits } \mid \text { 0x00 } \\ \text { Byte 6: } & \text { 0x00 } \\ \text { Bytes 7/8: } & \text { IP datagram length, big endian representation } \\ \text { Byte 9: } & 0 \times 00\end{array}$




\section{An Address Resolution Protocol}

When IP decides that the next hop for a datagram will be onto a SCSI network supported by a SCSI IP network interface implementation, it is necessary to acquire a data link address to deliver the datagram.

Network interfaces such as Ethernet have well-known methods for acquiring the media address for an Internet protocol address, the most common being the Address Resolution Protocol (ARP). In existing implementations, the forwarding host "yells" using a broadcast media address and expects the named host to respond.

The SCSI protocol does not provide a broadcast data link address. An acceptable solution to the address resolution problem for a SCSI network is to simulate a broadcast by performing a series of roundrobin transmissions to each target. Depending on the SCSI protocol being used, this would require upward of seven independent bus accesses. This is not grossly inefficient, however, if combined with an effective ARP caching policy. A further possible optimisation is negative ARP caching, whereby incomplete ARP bindings are not queried for some period in the future.

5. Scalability

While the utility of a network architecture based around a bus network which can span less than ten metres and support only eight hosts may be questionable, the flexibility of IP and in particular, IP routing, improves the scalability of this architecture.

Consider a network of eight hosts connected to a SCSI bus in which each host acts as a multi-homed host with a second host adapter connecting another seven hosts to it. When configured with IP packet routing capability, each of the 64 total hosts may communicate with one another at high speed in a packet switched manner.

Depending on the I/O bus capabilities of certain workstations, it may also be possible to configure a multi-homed host with a greater number of SCSI host adapters, permitting centralised star configurations to be constructed.

It should be apparent that for little expense, massively parallel virtual machines can be built based upon the IP protocol running over the high-bandwidth SCSI protocol. 
6. Possible Applications

Research has been made into the capability of "networks of workstations", and their performance compared to supercomputers. An observation that has been made thus far is that bottlenecks exist in the channels by which executable code is transported between hosts for execution. A very high-speed network architecture based around the Internet protocol would permit a seamless transition of existing application software to a high-bandwidth environment.

Other applications that have been considered are server clusters for fault-tolerant NFS, World-Wide Web and database services.

7. Security Considerations

Transmitting IP datagrams across a SCSI bus raises similar security issues to other local area networking architectures. The scale of security problems relating to protocols above the data link layer should be obvious to a reader current in Internet security.

8. References

[1] ANSI X3T9 Technical Committee, "Small Computer system Interface - 2", X3T9.2, Project 375D, Revision 10L, september 1993 .

9. Author's Address

Ben Elliston

Compucat Research Pty Limited

Box 7305 Canberra Mail Centre

Canberra ACT 2610

Australia

Phone: +6162951331

Fax: +6162951855

Email: ben.elliston@compucat.com.au 